\title{
Epileptic Focus Stimulation and Seizure Control in the Rat Model of Kainic Acid-Induced Limbic Seizures
}

\author{
Tomoyuki URINO, Kiyotaka HASHIZUME*, Michiyo MAEHARA**, Kouichi KATO, \\ Yoshikazu OKADA, Tomokatsu HORI, and Tatsuya TANAKA* \\ Departments of Neurosurgery and ${ }^{* *}$ Clinical Laboratory, \\ Tokyo Women's Medical University, Tokyo; \\ ${ }^{*}$ Department of Neurosurgery, Asahikawa Medical Collage, Asahikawa, Hokkaido
}

\begin{abstract}
The inhibitory effects of deep brain stimulation (DBS) were investigated in a rat model of kainic acid (KA)-induced limbic status epilepticus. Wistar rats were injected with $1.0 \mu \mathrm{g}$ KA into the left amygdala after stereotactic implantation of a guide cannula and electrodes. Bipolar rectangular pulses of $0.1 \mathrm{msec}$ duration and 0.1-0.3 $\mathrm{mA}$ amplitude were applied intermittently to the left amygdala $(10 \mathrm{~Hz}$ or $130 \mathrm{~Hz})$, left ventral hippocampus $(10 \mathrm{~Hz})$, and left dorsomedial thalamus $(130 \mathrm{~Hz})$. Seizure frequency was evaluated by video electroencephalography monitoring and compared to control animals that did not receive DBS. All rats developed limbic status epilepticus 60-90 minutes after KA injection. Seizure frequency was significantly reduced by $10 \mathrm{~Hz}$ stimulation of the amygdala and by $130 \mathrm{~Hz}$ stimulation of the dorsomedial thalamus. No significant effects were observed with other types of stimulation. Seizure behaviors or duration of seizure were not changed significantly by DBS treatment. DBS of an epileptic focus may attenuate KA-induced limbic seizures, depending on the stimulation sites and parameters.
\end{abstract}

Key words: deep brain stimulation, antiepileptic effect, experimental epilepsy, kainic acid, limbic seizure

\section{Introduction}

Surgical resection of the temporal lobe, hippocampus, or amygdala is effective for the treatment of intractable temporal lobe epilepsy. However, ablative surgeries require general anesthesia and craniotomy, and carry risks for postoperative complications, such as verbal memory decline following resection of the language-dominant hemisphere. ${ }^{9,11)}$ Deep brain stimulation (DBS) through stereotactic depth electrode implantation has gained recent attention as a novel and minimally invasive surgical therapy for intractable epilepsy.

DBS has been used clinically for the treatment of involuntary movement or intractable pain. High frequency stimulation of the ventral intermediate nucleus of the thalamus (VIM) is as effective as conventional ablative surgery in the treatment of Parkinson's disease. Stereotactic destruction of the deep brain structures was formerly performed for the treatment of intractable epilepsy. ${ }^{13)}$ DBS of the ventral lateral nucleus of the thalamus modified sei-

Received June 18, 2009; Accepted September 25, 2009 zure activity in experimentally-induced amygdala kindling, ${ }^{20)}$ and clinical application of electrical stimulation of the cerebellum has achieved favorable results. ${ }^{6)}$

Epilepsy treatment using electrical stimulation first involved stimulation of the cerebellum. ${ }^{4,5)} \mathrm{Va}$ gus nerve stimulation (VNS) has been applied clinically for epilepsy treatment, ${ }^{1,15}$ and recent progress in subthalamic nucleus stimulation has allowed clinical use for the treatment of intractable epilepsy. ${ }^{3,16)}$ However, subthalamic DBS may be ineffective for limbic seizures, although highly effective for focal cortical seizures. ${ }^{8}$ Direct electrical stimulation of epileptic foci has also been attempted to suppress epileptic seizures. Stimulation of a cortical epileptic focus with indwelling subdural electrodes, ${ }^{28)}$ hippocampal stimulation for the treatment of intractable temporal lobe epilepsy, and amygdalohippocampal stimulation for epilepsy treatment ${ }^{24}$ have all been reported with successful results.

Neuronal activity at the stimulation site is increased by low frequency electrical stimulation and decreased by high frequency stimulation. ${ }^{7)}$ Thus, the inhibitory effects of high frequency DBS can be attributed to functional ablation of neuronal activity 
in the target region. Given the inhibitory effects of electrical stimulation on neuronal activity, we predicted that electrical stimulation of an epileptic focus using adequate stimulation parameters would suppress abnormal discharges in the region. In the present study, we examined the inhibitory effects of direct electrical stimulation of an epileptic focus and its propagation pathways on experimentally-induced limbic seizures.

\section{Materials and Methods}

Forty-seven male Wistar rats (260-340 g body weight) were used in the limbic seizure model, including 12 rats as controls. Four different DBS procedures were conducted: Low frequency $(10 \mathrm{~Hz})$ stimulation of the basolateral amygdala $(n=9)$, high frequency $(130 \mathrm{~Hz})$ stimulation of the basolateral amygdala ( $\mathrm{n}=7), 10-\mathrm{Hz}$ stimulation of the ventral hippocampus ( $\mathrm{n}=9$ ), and $130-\mathrm{Hz}$ stimulation of the dorsomedial thalamus $^{14)}$ (MD) $(\mathrm{n}=10)$. All stimulation sites were ipsilateral to the seizure focus (left amygdala).

Under sodium pentobarbital anesthesia $(50 \mathrm{mg} / \mathrm{kg}$ intraperitoneally), animals were placed on a stereotactic instrument, the scalp was shaved and sterilized, a midline skin incision was made, and the calvaria was exposed. Burr holes were made using an electric drill, and a stainless steel cannula (diameter $0.6 \mathrm{~mm}$ ) was inserted into the left amygdala (coordinates: anterior $=+5.0 \mathrm{~mm}$, lateral $=+5.0$ $\mathrm{mm}$, depth $=-3.0 \mathrm{~mm}$ ) according to the atlas of Pellegrino et al. ${ }^{17)} \mathrm{A}$ Teflon-insulated stainless steel bipolar electrode was placed on a electrode manipulator and inserted into each target region with the tips separated by $0.9 \mathrm{~mm}$ anteroposteriorly. Depth electrodes for electroencephalography (EEG) recording were inserted into the bilateral amygdalas (at a 30-degree angle caudally for the left amygdala). Stainless steel screw electrodes were implanted in the frontal sinus and occipital bone as reference and ground electrodes, respectively. The cannula and all electrodes were connected to a socket and secured onto the skull with dental resin. At least 7 days were allowed to pass before behavioral experiments were performed, in order to ensure recovery from surgery.

Test stim ulations were performed before seizure induction by kainic acid (KA) injection using bipolar rectangular pulses of $0.1 \mathrm{msec}$ duration, which were selected to avoid cell damage. Current was set to $50-70 \%$ of the threshold amplitude $(0.1-0.3 \mathrm{~mA}$, mean $0.2 \mathrm{~mA}$ ) which induced abnormal behavior, or dyskinesia in the case of MD stimulation. Without anesthesia or physical restraint, a stainless steel in- jection needle with an external diameter of $0.3 \mathrm{~mm}$ was inserted into the left amygdala through the guide cannula, and $1 \mu \mathrm{g} \mathrm{KA}(1 \mu \mathrm{g} / \mu \mathrm{l})$ in $0.1 \mathrm{M}$ phosphate buffer ( $\mathrm{pH} 7.4)$ was injected slowly over a 1minute period, using a microsyringe. The injection needle was left in the cannula for at least 2 minutes after completion of the injection to allow diffusion of KA. Limbic status epilepticus was observed in all animals 1-1.5 hours after KA injection. ${ }^{21)}$ EEG and the behavior of the animals were recorded continuously by video EEG monitoring for 6.5 hours after KA injection. Intermittent DBS of the amygdala, ventral hippocampus, or MD was initiated 2.5 hours after KA injection during limbic status epilepticus. DBS for a 40-minute period, followed by a 20-minute interruption, was repeated 4 times until 6.5 hours after KA treatment, by which time the limbic status epilepticus had attenuated. The frequency, duration, and secondary generalization of seizures were evaluated based on the video EEG monitoring.

After completion of the experiments, all animals were perfused with saline followed by $4 \%$ paraformaldehyde, under deep anesthesia. The brain was dissected for histological analysis to confirm the precise placement of the guide cannula for KA injection in the left amygdala and stimulating electrodes in each target region.

\section{Results}

All animals developed limbic seizures 10-30 minutes after KA injection. The limbic seizures were characterized by akinesia accompanied by salivation and mydriasis, followed by unilateral facial clonus and masticatory movement, with EEG findings of multiple spike complexes in the amygdala ipsilateral to the KA injection. Multiple spike complexes were detected in bilateral amygdalas after 30 minutes to 1 hour (Fig. 1), and the animals displayed rolling to the side contralateral to the KA injection, with occasional rearing and falling with foreleg clonus. Generalized tonic-clonic seizures with salivation were occasionally observed, most of which led to secondary generalization. Seizure was defined as an event with consistent EEG findings and video EEG monitoring behavior. Seizures occurred once every 2-4 minutes after 1-1.5 hours, suggesting the presence of acute limbic status epilepticus. DBS was initiated 2.5 hours after KA injection, and the number of seizures was counted for the period between 1.5 and 6.5 hours.

Seizure frequency of KA-induced limbic status epilepticus attenuated spontaneously 1.5 hours after injection in the control animals, but such attenuation was apparently enhanced by $10-\mathrm{Hz}$ DBS in the 
amygdala (Fig. 2A). Analysis of variance was performed using the general linear model procedure in SAS Institute (Cary, N.C., U.S.A.), to compare seizure frequency between animals receiving DBS and controls, as a function of time. Seizure frequency was significantly lower ( $p<0.05)$ in the DBS group during the second DBS, and even during the subsequent interruption of DBS. Therefore, seizure sup-

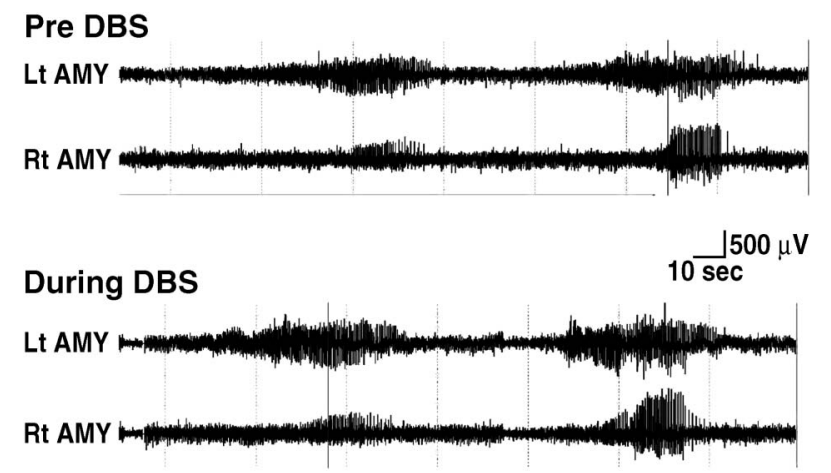

Fig. 1 Electroencephalograms recorded before and during $10-\mathrm{Hz}$ deep brain stimulation (DBS) of the left amygdala showing multiple spike complexes in the bilateral amygdalas, with initiation in the left amygdala for each seizure. Seizure duration is approximately 1 minute. No apparent changes were noted during DBS. Lt AMY: left amygdala, Rt AMY: right amygdala.

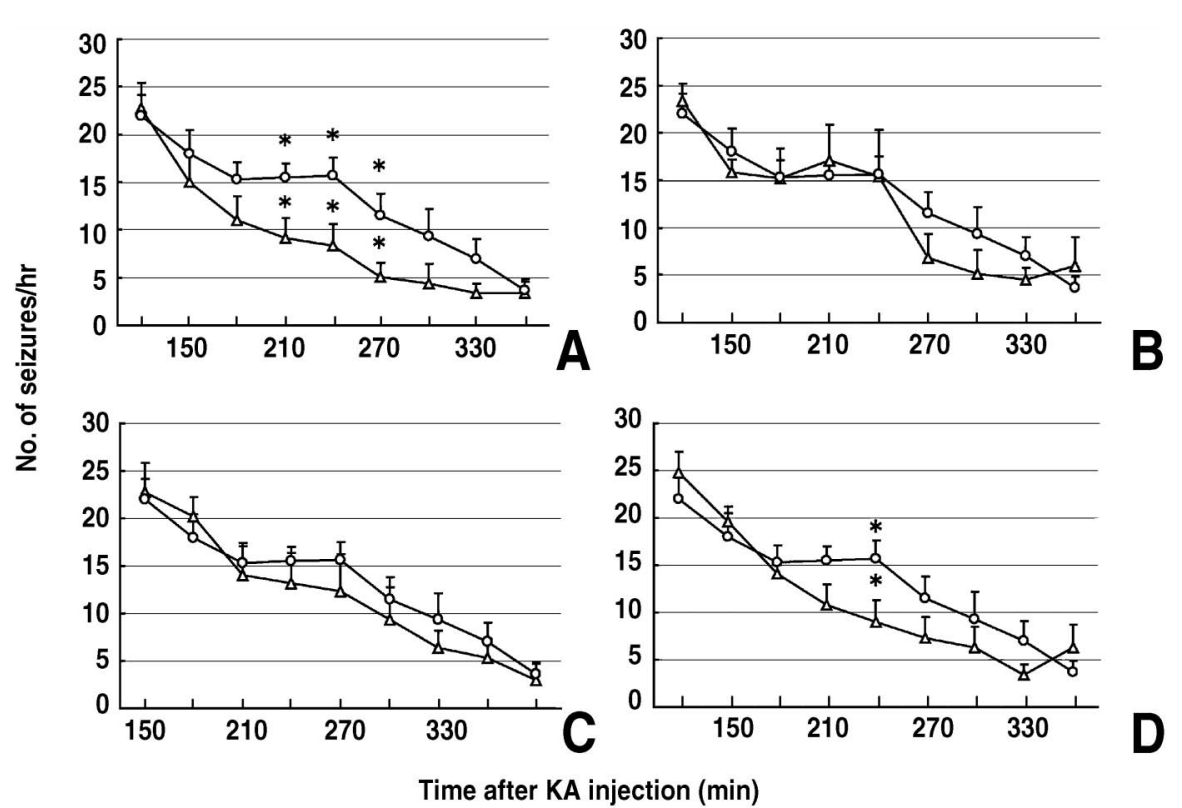

Fig. 2 Time course of seizure frequency and effects of deep brain stimulation (DBS). A: Significantly lower seizure frequency was observed during the period between the second 10-Hz DBS of the amygdala, 210 minutes after kainic acid (KA) injection, and third DBS, compared to controls. B, C: 130-Hz DBS of the amygdala (B) and 10-Hz DBS of the hippocampus (C) showed no significant differences with the controls. D: Seizure frequency was significantly reduced during the second interruption of $130-\mathrm{Hz}$ DBS of the dorsomedial thalamus. ${ }^{*} p<0.05$, General Linear Model procedure. $\triangle$ : amygdala $10-\mathrm{Hz}(\mathrm{n}=9)$, amygdala $130-\mathrm{Hz}(\mathrm{n}=7)$, hippocampus $10-\mathrm{Hz}(\mathrm{n}=9)$, or dorsomedial thalamus 130-Hz $(n=10)$ stimulation group; $\bigcirc$ : controls $(n=12)$.

pression was retained during the interruption of DBS. The difference remained significant during the third DBS ( $p<0.05$ ). However, seizure frequency in the control animals also decreased with time and reached levels almost equal to those in the DBS group by 6.5 hours after KA treatment. High frequency DBS $(130 \mathrm{~Hz})$ has different functional properties to low frequency DBS $(10 \mathrm{~Hz})($ Fig. 2B). Seizure frequency was not significantly different between animals receiving $130 \mathrm{~Hz}$ DBS in the amygdala and the controls at any time point.

The ventral hippocampus, which is closest to the amygdala along the seizure propagation pathway, was stimulated by $10-\mathrm{Hz}$ DBS with the same method as that used for amygdala stimulation. No significant effects of DBS treatment were observed compared to the controls at any time point (Fig. 2C).

The MD, which receives inputs from the limbic system, was stimulated by $130 \mathrm{~Hz}$ DBS. Statistically significant suppression $(p<0.05)$ of seizures was detected only during the interruption period following the second DBS (Fig. 2D). No significant effects of DBS were observed during the subsequent stages.

Most seizures were associated with multiple spike complexes in the bilateral amygdalas and with secondary generalization. All types of DBS treatment failed to attenuate seizure EEG, and failed to suppress secondary generalization. The duration of 
mono- and bipolar electrodes
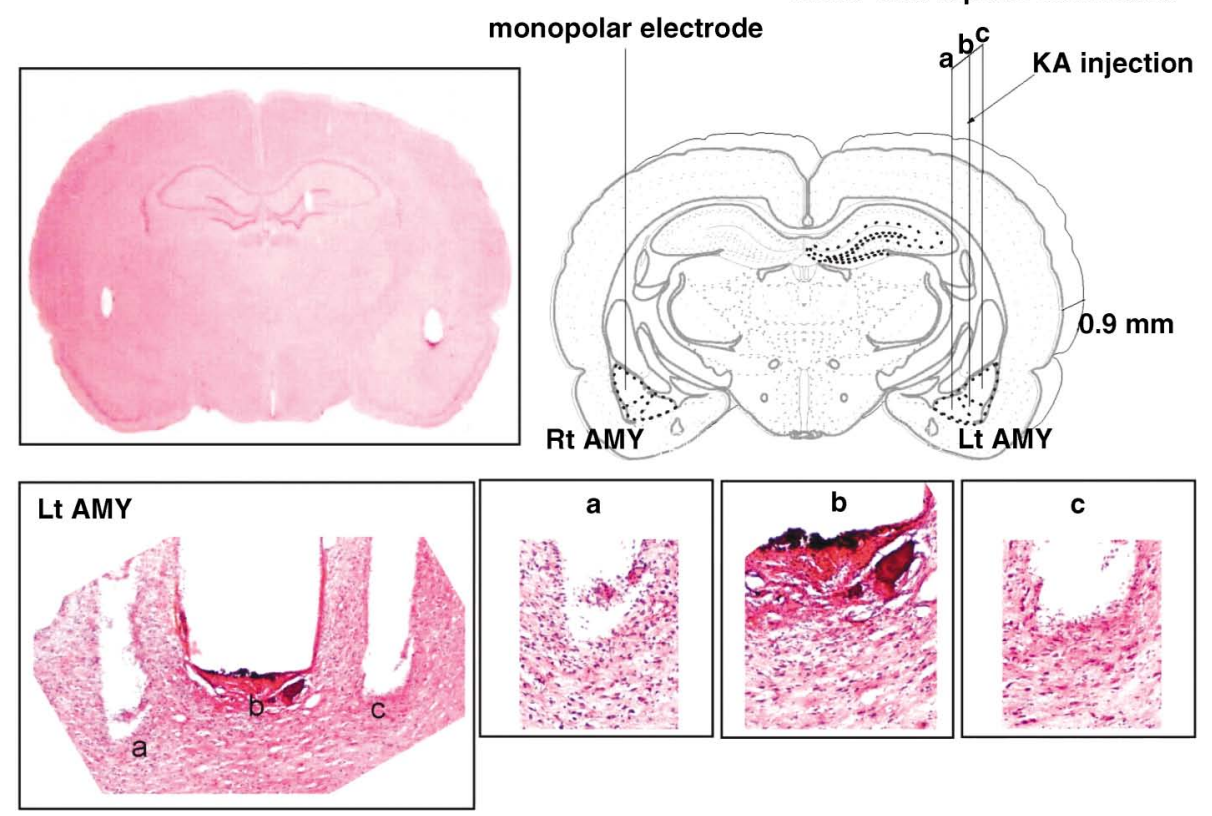

Fig. 3 Photomicrographs showing the sites of the guide cannula for kainic acid (KA) injection (1.0 $\mu$ g) inserted directly above the left amygdala (Lt AMY), with the tip of the injection needle located $0.5 \mathrm{~mm}$ below the tip of the guide cannula, and localization of the tips of electrodes in the right amygdala (Rt AMY). Upper row: axial sections, lower row: sagittal sections. Electrodes a and c: tips of the stainless steel bipolar electrodes, b: tip of the stainless steel cannula (diameter $0.6 \mathrm{~mm}$ ).

each seizure, as measured by EEG, ranged widely from 15 to 150 seconds, and no effects of DBS on seizure duration were observed.

Precise placement of the guide cannula for KA injection in the left amygdala and stimulating electrodes in each target region was confirmed by histological examination. KA-induced necrotic lesion 1-2 $\mathrm{mm}$ in diameter was observed around the injection site in the amygdala, as reported previously. ${ }^{13)}$ No morphological changes were identified in structures around the site of electrical stimulation (Fig. 3).

\section{Discussion}

The mechanism underlying the induction of convulsive seizures by intracerebral, intraventricular, intravenous, and intraperitoneal administration of excitatory amino acids is ascribed to excessive neuronal activity mediated by glutamate receptor activation. Systemic administration generally induces seizures with bilateral epileptic foci or generalization. Convulsive seizures evoked by microinjection of KA into the unilateral amygdala in rats provides an excellent model of epilepsy which resembles temporal lobe epilepsy in humans in terms of symptoms, electrophysiology, pathology, brain metabolism, and cerebral blood flow (CBF).,21) Local KA injection causes excessive neuronal activity at and around the injection site, which propagates by physiologic pathways to induce convulsive seizures. In the present study, we used a relatively high dose of KA $(1.0 \mu \mathrm{g})$ to create a particularly severe model of limbic status epilepticus. Therefore, our finding of attenuation of seizures by DBS of the epileptic focus is particularly significant in this model.

In the present model of acute limbic status epilepticus, the inhibitory effects of DBS depended on the site and parameters of stimulation. Low frequency stimulation of the seizure focus was most effective. Seizure propagation can be suppressed by transection surgery between the dorsal and ventral hippocampus in a KA-induced rat epilepsy model. ${ }^{12}$ However, hippocampal blockade by DBS in our study failed to reduce the frequency of seizures. Bilateral hippocampal lesioning by ibotenic acid injection delays the development of amygdala kindling, but fails to prevent secondary generalization of the seizure. ${ }^{19)}$

Seizure frequency was reduced by DBS of the MD, possibly by blocking the propagation pathway of amygdala seizure. However, DBS of the hippocampus did not affect the seizures, despite the fact that amygdala seizure first propagates to the hippocampus. Seizure frequency was reduced by DBS of the epileptic focus, but no changes in EEG findings were observed. Therefore, the effects of DBS on 
epilepsy may be mediated by the inhibition of electrophysiological changes which trigger the onset of seizures.

DBS of the basal ganglia has different effects on brain functions, depending on the stimulation frequency. DBS of the VIM for the treatment of essential tremor showed that an increase in stimulation frequency from $130 \mathrm{~Hz}$ to $180 \mathrm{~Hz}$ caused a linear increase in regional CBF ( $\mathrm{rCBF}$ ) in the primary sensorimotor cortex, but a nonlinear $\mathrm{rCBF}$ response at the stimulation site in the VIM, as revealed by positron emission tomography. ${ }^{22}$ The reverse pattern in rCBF responses was observed by increasing the stimulation amplitude from $1.5 \mathrm{~V}$ to $3.0 \mathrm{~V}$. These results suggest close connectivity between the VIM and primary sensorimotor cortex, and stimulation parameter-specific modulation of the cerebral system. ${ }^{10)}$ The present study demonstrated that low frequency stimulation had inhibitory effects in the limbic system, whereas high frequency stimulation was effective in the MD. Subacute and chronic electrical stimulation of the hippocampus at $130 \mathrm{~Hz}$ is effective for the treatment of temporal lobe epilepsy in humans, ${ }^{23)}$ whereas low frequency $(1 \mathrm{~Hz})$ stimulation of the epileptic focus has inhibitory effects on amygdala kindling in rats. ${ }^{26,27)}$ Therefore, the effects of varying stimulation parameters for DBS remain controversial.

The present study observed statistically significant inhibition of seizure frequency 60 minutes after onset of $10-\mathrm{Hz}$ amygdala stimulation and 100 minutes after $130 \mathrm{~Hz}$ MD stimulation, suggesting delayed inhibitory effects of DBS on epileptic seizures. VNS induces rCBF changes in the thalamus immediately after stimulation, as revealed by singlephoton emission computed tomography, ${ }^{25)}$ but the maximal inhibition of experimentally-induced seizures occurs after 60 minutes of continuous VNS, 18) also suggesting delayed effects of VNS. Changes in brain hemodynamics induced by hippocampal, amygdalar, and thalamic inhibition have been implicated in the long-term effects of VNS.22) The mechanism underlying the effects of DBS of the amygdala remains to be investigated.

Insertion of depth electrodes does not require resection of brain tissue, so is less invasive than surgical removal of the epileptic focus. In addition, the stimulating electrodes can be removed when they become unnecessary, so clinical application of DBS is desirable. However, simultaneous stimulation of multiple brain regions may be required to effectively control the clinical symptoms of epileptic seizures, and the synergistic effects of DBS combined with antiepileptic agents or surgical therapy must be studied. The long-term inhibitory effects of DBS on epileptic seizures also remain to be explored.

\section{Acknowledgments}

The authors thank Dr. Makoto Ozaki (Department of Anesthesiology, Tokyo Women's Medical University) for providing experimental equipment, Dr. Satoru Shimizu (Medical Research Institute, Tokyo Women's Medical University) for assistance with statistical analysis.

\section{References}

1) Annegers JF, Coan SP, Hauser WA, Leestma J, Duffell W, Tarver B: Epilepsy, vagal nerve stimulation by the NCP system, mortality, and sudden, unexpected, unexplained death. Epilepsia 39: 206-212, 1998

2) Ben-Ari Y: Limbic seizure and brain damage produced by kainic acid: Mechanisms and relevance to human temporal lobe epilepsy. Neuroscience 14: 375-403, 1985

3) Bingaman WE, Hadar EJ, Najim IM: Chronic stimulation of the subthalamic nucleus for the treatment of medically-intractable seizures: a case report. Epilepsia 41 Suppl 7: 149, 2000

4) Cooper IS: Effect of chronic stimulation of anterior cerebellum on neurological disease. Lancet 1(7796): 206, 1973

5) Cooper IS, Amin I, Gilman S: The effect of chronic cerebellar stimulation upon epilepsy in man. Trans Am Neurol Assoc 98: 192-196, 1973

6) Cooper IS, Upton AR: Therapeutic implications of modulation of metabolism and functional activity of cerebral cortex by chronic stimulation of cerebellum and thalamus. Biol Psychiatry 20: 811-813, 1985

7) Depaulis A, Vergnes M, Marescaux C: Endogenous control of epilepsy: the nigral inhibitory system. Prog Neurobiol 42: 33-52, 1994

8) Deransart C, Lê BT, Marescaux C, Depaulis A: Role of the subthalamo-nigral input in the control of amygdala-kindled seizures in the rat. Brain Res 807: 78-83, 1998

9) Gleissner U, Helmstaedter C, Schramm J, Elger CE: Memory outcome after selective amygdalohippocampectomy: a study in 140 patients with temporal lobe epilepsy. Epilepsia 43: 87-95, 2002

10) Haslinger B, Boecker H, Buchel C, Vesper J, Tronnier VM, Pfister R, Alesch F, Moringlane JR, Krauss JK, Conrad B, Schwaiger M, Ceballos-Baumann AO: Differential modulation of subcortical target and cortex during deep brain stimulation. Neuroimage 18: 517-524, 2003

11) Hermann BP, Wyler AR, Bush AJ, Tabatabai FR: Differential effects of left and right anterior temporal lobectomy on verbal learning and memory performance. Epilepsia 33: 289-297, 1992

12) Imamura $S$, Tanaka $S$, Akaike $K$, Tojo $H$, Takigawa M, Kuratsu J: Hippocampal transection attenuates kainic acid-induced amygdalar seizures in rats. Brain Res 897: 93-103, 2001

13) Jinnai D, Nishimoto A: Stereotaxic destruction of Forel-H for treatment of epilepsy. Neurochirurgia (Stuttg) 25: 164-176, 1963

14) Kato K, Urino T, Hori T, Tsuda H, Yoshida K, Hashi- 
zume K, Tanaka T: Experimental petit mal-like seizure induced by microinjection of kainic acid into the unilateral mediodorsal nucleus of the thalamus. Neurol Med Chir (Tokyo) 48: 285-291, 2008

15) Labar D, Nikolov B, Tarver B, Fraser R: Vagus nerve stimulation for symptomatic generalized epilepsy: a pilot study. Epilepsia 39: 201-205, 1998

16) Loddenkemper T, Pan A, Neme S, Baker B, Rezai AR, Dinner DS, Montgomery EB Jr, Lüders HO: Deep brain stimulation in epilepsy. J Clin Neurophysiol 18: 514-532, 2001

17) Pellegrino LJ, Pellegrino AS, Cushman AJ: A Stereotaxic Atlas of the Rat Brain, $2^{\text {nd }}$ ed. New York, Plenum Press, 1979

18) Takaya M, Terry WJ, Naritoku DK: Vagus nerve stimulation induces a sustained anticonvulsant effect. Epilepsia 37: 1111-1116, 1996

19) Tanaka T, Kondo S, Hori T, Tanaka S, Yonemasu Y: Various hippocampal lesions induced by multi-fractional ibotenic acid injections and amygdala kindling in rats. Brain Res 559: 154-158, 1991

20) Tanaka T, Lange H, Naquet R: Sleep, subcortical stimulation and kindling in the cat. Can J Neurol Sci 2: 447-455, 1975

21) Tanaka T, Tanaka S, Fujita T, Takano K, Fukuda H, Sako K, Yonemasu Y: Experimental complex partial seizures induced by a microinjection of kainic acid into limbic structures. Prog Neurobiol 38: 317-334, 1992

22) Van Laere K, Vonck K, Boon P, Versijpt J, Dierckx R: Perfusion SPECT changes after acute and chronic vagus nerve stimulation in relation to prestimulus condition and long-term clinical efficacy. J Nucl Med 43: 733-744, 2002

23) Velasco AL, Velasco M, Velasco F, Menes D, Gordon F, Rocha L, Briones M, Márquez I: Subacute and chronic electrical stimulation of the hippocampus on intractable temporal lobe seizures: preliminary report. Arch Med Res 31: 316-328, 2000

24) Vonck K, Boon P, Achten E, De Reuck J, Caemaert J: Long-term amygdalohippocampal stimulation for refractory temporal lobe epilepsy. Ann Neurol 52: 556-565, 2002

25) Vonck K, Boon P, Van Laere K, D’Havé M, Vandekerckhove T, O’Connor S, Brans B, Dierckx R, De Reuck $\mathrm{J}$ : Acute single photon emission computed tomographic study of vagus nerve stimulation in refractory epilepsy. Epilepsia 41: 601-609, 2000

26) Weiss SR, Eidsath A, Li XL, Heynen T, Post RM: Quenching revisited: low level direct current inhibits amygdala-kindled seizures. Exp Neurol 154: 185-192, 1998

27) Weiss SR, Li XL, Rosen JB, Li H, Heynen T, Post RM: Quenching: inhibition of development and expression of amygdala kindled seizures with low frequency stimulation. Neuroreport 6: 2171-2176, 1995

28) Yamamoto J, Ikeda A, Satow T, Takeshita K, Takayama M, Matsuhashi M, Matsumoto R, Ohara S, Mikuni N, Takahashi J, Miyamoto S, Taki W, Hashimoto N, Rothwell JC, Shibasaki H: Low frequency electric cortical stimulation has an inhibitory effect on epileptic focus in mesial temporal lobe epilepsy. Epilepsia 43: 491-495, 2002

Address reprint requests to: Tomoyuki Urino, M.D., Department of Neurosurgery, Tokyo Women's Medical University, 8-1 Kawada-cho, Shinjuku-ku, Tokyo 162-8666, Japan.

e-mail: turino@peach.ocn.ne.jp

\section{Commentary}

In the past decade, deep brain stimulation (DBS) has been investigated for its potential use in the treatment of epilepsy in several animal models and in patients with refractory epilepsy. Numerous studies targeting at different anatomic sites including the thalamus, subthalamic nucleus, cerebellum, hippocampus, caudate nucleus and mammillary nuclei demonstrate interesting results. However, the fundamental mechanisms of DBS on seizure reduction, the optimal stimulation parameters and the long term effects of DBS on seizure frequency remain largely unknown. The quest to find appropriate anatomic targets and stimulation protocols that can effectively modulate the level of epileptogenicity is an ongoing effort.

Urino and colleagues report that direct stimulation at the epileptogenic focus can produce a significant seizure reduction in animals with KA-induced limbic status epilepticus. In addition, they found that the effect of DBS varies with the stimulation sites and parameters used. Low frequency but not high frequency stimulation at the amygdala and high frequency stimulation at the dorsomedial thalamus showed a beneficial effect. The results of this study are interesting and provocative. A pilot study of eight patients using high frequency stimulation applied directly to the epileptogenic zone and indirectly through the anterior thalamic nucleus bilaterally reported an immediate improvement in seizure frequency. ${ }^{1)}$ More recently, Velasco and associates showed that stimulation at the amygdala and the anterior hippocampus was associated with a reduction in seizures by $50-70 \%$ in four patients with mesial temporal sclerosis. ${ }^{2)}$ It becomes quite clear that brain stimulation at the epileptogenic focus provides immediate beneficial effect in seizure reduction. However, the magnitude and long term effects of the seizure control remains questionable. Further explorations are needed to elucidate the potential mechanisms that underlie its effectiveness, to establish optimal parameter configurations, and to determine the long term effect in chronic refractory epilepsy.

\section{References}

1) Osorio I, Frei MG, Sunderam S, Giftakis J, Bhavaraju NC, Schaffner SF, Wilkinson SB: Automated seizure abatement in humans using electrical stimulation. Ann Neurol 57: 258-268, 2005

2) Velasco AL, Velasco F, Velasco M, Trejo D, Castro G, Carrillo-Ruiz JD: Electrical stimulation of the hippocampal epileptic foci for seizure control: a double-blind, long-term follow-up study. Epilepsia 48: 1895-1903, 2007

Kitti Kaiboriboon, M.D. and Hans O. LÜDERS, M.D., Ph.D. Epilepsy Center University Hospitals Case Medical Center Cleveland, Ohio, U.S.A. 